\title{
Terrestrial gamma ray flash production by lightning current pulses
}

\author{
B. E. Carlson, ${ }^{1}$ N. G. Lehtinen, ${ }^{1}$ and U. S. Inan ${ }^{1,2}$ \\ Received 4 June 2009; revised 28 July 2009; accepted 28 August 2009; published 19 December 2009.
}

[1] Terrestrial gamma ray flashes (TGFs) are brief bursts of gamma rays observed by satellites, typically in coincidence with detectable lightning. We incorporate TGF observations and the key physics behind current TGF production theories with lightning physics to produce constraints on TGF production mechanisms. The combined constraints naturally suggest a mechanism for TGF production by current pulses in lightning leader channels. The mechanism involves local field enhancements due to charge redistribution in current pulses and draws seed energetic particles from cold runaway in breakdown processes. The mechanism can reproduce the observed TGF time scale including multipulse TGFs, is in agreement with existing production altitude estimates, and may help explain TGF spectra by naturally producing unbeamed emissions.

Citation: Carlson, B. E., N. G. Lehtinen, and U. S. Inan (2009), Terrestrial gamma ray flash production by lightning current pulses, J. Geophys. Res., 114, A00E08, doi:10.1029/2009JA014531.

\section{Introduction}

[2] Terrestrial gamma ray flashes (TGFs) are brief bursts of energetic photons observed by satellites. Discovered in 1994 by the Burst And Transient Source Experiment on board the Compton Gamma Ray Observatory (BATSE/CGRO) [Fishman et al., 1994; Nemiroff et al., 1997], TGFs have since been observed in detail by the Reuven Ramaty High Energy Solar Spectroscopic Imager (RHESSI) [Smith et al., 2005; Grefenstette et al., 2009]. TGFs have a fluence of $\sim 1$ photon $/ \mathrm{cm}^{2}$, a time scale of $\sim 0.5 \mathrm{~ms}$ and photon energies of up to $20 \mathrm{MeV}$ when seen from orbit [Smith et al., 2005; Grefenstette et al., 2008]. TGFs are also usually observed in close time coincidence with lightning discharge detectable in radio measurements [Inan et al., 2006; Cohen et al., 2006; Cummer et al., 2005; Stanley et al., 2006]. These observations pose an interesting question: how are so many photons produced with such high energy in such a short time scale in coincidence with lightning? We examine this question by first surveying TGF observations and what constraints they place on the TGF source. Second, we give a broad survey of lightning physics and the constraints it places on TGF production. The combination of these constraints suggests a plausible TGF production mechanism: current pulses along developing lightning leader channels. We then examine this mechanism in the context of TGF and

\footnotetext{
${ }^{1}$ Space, Telecommunications and Radioscience Laboratory, Electrical Engineering Department, Stanford University, Stanford, California, USA.

${ }^{2}$ Department of Electrical and Electronics Engineering, Koç University, Istanbul, Turkey.

Copyright 2009 by the American Geophysical Union. 0148-0227/09/2009JA014531
}

lightning observations and make predictions for future experiments.

\section{Survey of TGF Physics}

[3] TGFs are $\sim 0.5 \mathrm{~ms}$ pulses of photons with energies $\mathcal{E} \gamma$ that can exceed $20 \mathrm{MeV}$ [Fishman et al., 1994; Smith et al., 2005]. Radio observations of lightning indicate that these flashes are typically detected within a few milliseconds of lightning discharge [Inan et al., 2006; Cohen et al., 2006; Cummer et al., 2005; Stanley et al., 2006]. Though not all TGFs are accompanied by clear radio activity, the imperfect detection efficiency of such radio observations does not rule out lightning activity in these cases [Inan et al., 2006; Cohen et al., 2006]. When coincident lightning can be geolocated, it is typically less than $300 \mathrm{~km}$ from the subsatellite point with no noticed systematic offset. TGF fluence approaches $\sim 1$ photon $/ \mathrm{cm}^{2}$, though the absolute brightness is uncertain due to satellite dead-time issues [Grefenstette et al., 2008]. Typically, isolated $\sim 0.5 \mathrm{~ms}$ pulses are detected, but groups of pulses separated by several milliseconds are sometimes observed [Fishman et al., 1994]. TGFs are observed by the RHESSI instrument every $\sim 2$ days [Smith et al., 2005]. Though the global frequency of TGFs is difficult to determine due to efficiency concerns (trigger efficiency, dead time, limited effective area and energy response), assuming the $300 \mathrm{~km}$ maximum subsatellite-lightning distance is the maximum detection distance, this implies the global TGF frequency is $\geq 500 / \mathrm{d}$.

[4] We now consider key known facts about TGFs. The first key fact is the $\mathcal{E} \gamma>20 \mathrm{MeV}$ energy scale. Populations of such photons must be produced by bremsstrahlung. Monte Carlo simulations of RREA and bremsstrahlung executed with the GEANT4 software package [Agostinelli et al., 2003] indicate that the population of electrons is typically $\sim 10$ times larger than the population of photons. 
The close time and subsatellite-lightning position coincidence suggest that electric fields associated with lightning produce or accelerate these electrons and requires that the electric fields cover a potential difference of at least $20 \mathrm{MV}$.

[5] The second key fact is the $\sim 1$ photon $/ \mathrm{cm}^{2}$ fluence at satellite altitude. Assuming TGF photons are distributed uniformly over a disk in the satellite plane with radius given by the typical subsatellite-lightning distance $(300 \mathrm{~km}$, cited above), one can calculate that $\sim 10^{15}$ photons reach satellite altitude. Losses as the photons escape the atmosphere require a larger initial population of photons and a correspondingly larger population of relativistic electrons.

[6] Moderate electric fields in air can naturally produce a large population of relativistic electrons by an avalanche growth process, referred to as runaway relativistic electron avalanche (RREA) [Wilson, 1924; Roussel-Dupré et al., 1994; Gurevich and Zybin, 2001]. To summarize, relativistic electrons gain energy from the electric field and lose energy to inelastic scattering. Scattering sometimes produces relativistic secondary electrons that then join in the process, increasing the population of relativistic electrons until the population exits the field region. RREA can occur at electric fields an order of magnitude smaller than the conventional dielectric breakdown threshold, and is therefore especially relevant to thunderstorm physics where E fields large enough to initiate breakdown are seldom observed [Betz et al., 2009]. The main complication is that RREA at moderate electric fields needs a source of relativistic electrons to seed the population. In the Earth's atmosphere these seed electrons come mostly from cosmic rays [Carlson et al., 2008]. Seed particles may also be directly produced by "cold runaway" in very strong electric fields $(E>26 \mathrm{MV} / \mathrm{m})$ [Moss et al., 2006]. Further, if the field region is very large $(U>50-100 \mathrm{MV}$, e.g., $100 \mathrm{~m}$ long, $E \sim 1 \mathrm{MV} / \mathrm{m})$, one population at low potential may be able to seed subsequent populations at high potential in a process dubbed "relativistic feedback" [Dwyer, 2003; Babich et al., 2005]. We discuss the source of seed electrons in detail in section 5 .

[7] The third key fact is the $\sim 0.5 \mathrm{~ms}(150 \mathrm{~km} / \mathrm{c})$ time scale, with TGF durations observed ranging from $0.2 \mathrm{~ms}$ to several milliseconds. As TGF photons arrive at satellite altitude with less than $\sim 100 \mu$ s dispersion [Østgaard et al., 2008; Grefenstette et al., 2008], the source time scale must closely match the observed photon time scale. We discuss this fact further in section 5 .

[8] Several mechanisms have been proposed to explain these key facts, differing mainly in the source of the electric field used to drive RREA. Early mechanisms assumed that TGFs originated at $30-80 \mathrm{~km}$ altitude as photons emitted at lower altitudes are less able to escape the atmosphere. These mechanisms used lightning-driven charge dipole moment changes [Lehtinen et al., 1996, 1999; Babich et al., 2004] and electromagnetic pulses [Inan and Lehtinen, 2005] to produce fields at high altitudes. Such mechanisms require extreme lightning parameters not found in existing observations in order to produce an observable number of photons (e.g., return stroke velocity $\geq 0.99 \mathrm{c},>500 \mathrm{kA}$ peak current, accounting for at most 6-12 TGFs per day [see Inan and Lehtinen, 2005]). Furthermore, detailed comparison of averaged photon spectra observed by RHESSI and BATSE with predicted spectra from RREA mechanisms indicate a much lower production altitude of $15-20 \mathrm{~km}$ for at least the majority of the events [Dwyer and Smith, 2005; Carlson et al., 2007]. These lower altitudes are consistent with tropical thunderstorm cloud tops [Williams et al., 2006], suggesting TGFs are produced in or very near the cloud and therefore in direct association with lightning and thunderstorm electric fields. Photons produced at these production altitudes are attenuated by a factor of 10-30 in number as they escape to satellite altitude [Carlson et al., 2007]. A population of $10^{15}$ escaping photons therefore requires $10^{16}$ photons at $15-20 \mathrm{~km}$ as would be produced by $10^{17}$ energetic electrons.

[9] In summary, TGFs require populations of $\sim 10^{17}$ electrons with energies up to and exceeding $20 \mathrm{MeV}$. These large populations of electrons are likely produced at or near cloud top altitude by RREA growth driven directly by thunderstorm and lightning electric fields covering potential differences of at least $20 \mathrm{MV}$. Whether and how such production might occur depends on thunderstorm and lightning physics.

\section{Survey of Relevant Lightning Physics}

[10] A complete review of lightning physics is far beyond the scope of this paper. Interested readers can find excellent reviews by Rakov and Uman [2003], Cooray [2004], Bazelian and Raizer [1998], and Gallimberti et al. [2002]. Overall, lightning is a branching network of narrow conducting plasma channels (leaders). Thunderstorm electric fields drive currents in the leader channels that redistribute charge in the cloud. The detailed physics of the formation and growth of the network is not well understood but involves a progression in scale from millimeterscale propagating ionization fronts (streamers) to meter-scale volumes with many streamers (corona), which heat the air sufficiently to produce the plasma in the leader channel. Overall, lightning involves charge redistribution. Charges flow through leader channels, pile up on leader tips, and are collected and dispersed near leader channels by streamer and corona discharge. These processes have been studied by a wide array of experimental and theoretical techniques and are described in detail in the reviews cited above. In this paper we simply give the parameters of the various processes in lightning discharge and describe how those parameters may lead to TGF production. These parameters are summarized in Table 1.

[11] The parameters relevant to TGF production are the magnitude of the electric field and the voltage available in the region spanned by the electric field. The electric field determines the possibility of production or growth of populations of relativistic electrons, while the voltage relates to the production of sufficiently energetic electrons. Lightning physics sets limits on these parameters and therefore provides constraints on possible TGF production mechanisms.

\section{Constraints}

[12] These constraints can be easily visualized if the voltage and electric field parameters are plotted together as shown in Figure 1. The minimum electric fields for RREA, conventional dielectric breakdown, and cold 
Table 1. Typical Parameters of Lightning Processes ${ }^{\mathrm{a}}$

\begin{tabular}{|c|c|}
\hline Process & Properties \\
\hline \multicolumn{2}{|l|}{ Streamers } \\
\hline $\begin{array}{l}\text { Tip } \\
+/- \text { propagation }\end{array}$ & $\begin{array}{c}q \sim 10^{-10} \mathrm{C}, E \sim 10 \mathrm{MV} / \mathrm{m}, l \sim 0.2 \mathrm{~mm}, U \sim 10-100 \mathrm{kV} \\
E=0.5 / 1.5 \mathrm{MV} / \mathrm{m}\end{array}$ \\
\hline \multicolumn{2}{|r|}{ 然 } \\
\hline Tip/step & $q \sim 10-100 \mathrm{mC}, r \sim 6 \mathrm{~m}, l \sim 10-100 \mathrm{~m}, U \sim 20-50 \mathrm{MV}$ \\
\hline Channel & $\lambda \sim 0.7-30 \mathrm{mC} / \mathrm{m}, r \sim 6 \mathrm{~m}, l \sim \mathrm{km}, q_{\mathrm{tot}} \sim 10 \mathrm{C}, U \sim 20-50 \mathrm{MV}$ \\
\hline Propagation & $E \sim 0.1 \mathrm{MV} / \mathrm{m}$ \\
\hline \multicolumn{2}{|l|}{ Lightning } \\
\hline Leader system & $q \sim 10 \mathrm{C}, E \sim 0.1 \mathrm{MV} / \mathrm{m}, l \sim 500 \mathrm{~m}, U \sim 10 \mathrm{MV}$ \\
\hline Return stroke & $q \sim 10 \mathrm{C}$ \\
\hline Overall & $q \sim 100 \mathrm{C}, E \sim 0.1 \mathrm{MV} / \mathrm{m}, l \sim$ few $\mathrm{km}, U \sim 50 \mathrm{MV}$ \\
\hline \multicolumn{2}{|r|}{1} \\
\hline Overall & $q \sim 100 \mathrm{C}, E \sim 0.1 \mathrm{MV} / \mathrm{m}, l \sim$ few $\mathrm{km}, U \sim 100 \mathrm{MV}$ \\
\hline
\end{tabular}

runaway are shown on the electric field axis. The maximum voltage available in a typical thunderstorm $(\sim 100 \mathrm{MV}$, though this number is difficult to measure [see Marshall and Stolzenburg, 2001]), a 20 MV reference potential, and the minimum voltage necessary to accelerate an energetic electron to $20 \mathrm{MeV}$ with friction included are also shown. The shaded region of the plot satisfies the constraints from TGF production while also falling in the region allowed by thunderstorm maximum voltage.
[13] The lightning processes listed above can be analyzed to estimate the typical average electric field and available voltage. Here we consider simple geometries for the charge structures: point, line, or plane. Since no true point or line charges can exist, we assume the "point" and "line" geometries are actually uniform spherical and cylindrical volumes with charge distributed uniformly out to the smallest radius such that the electric field never exceeds the conventional breakdown threshold. Since we are

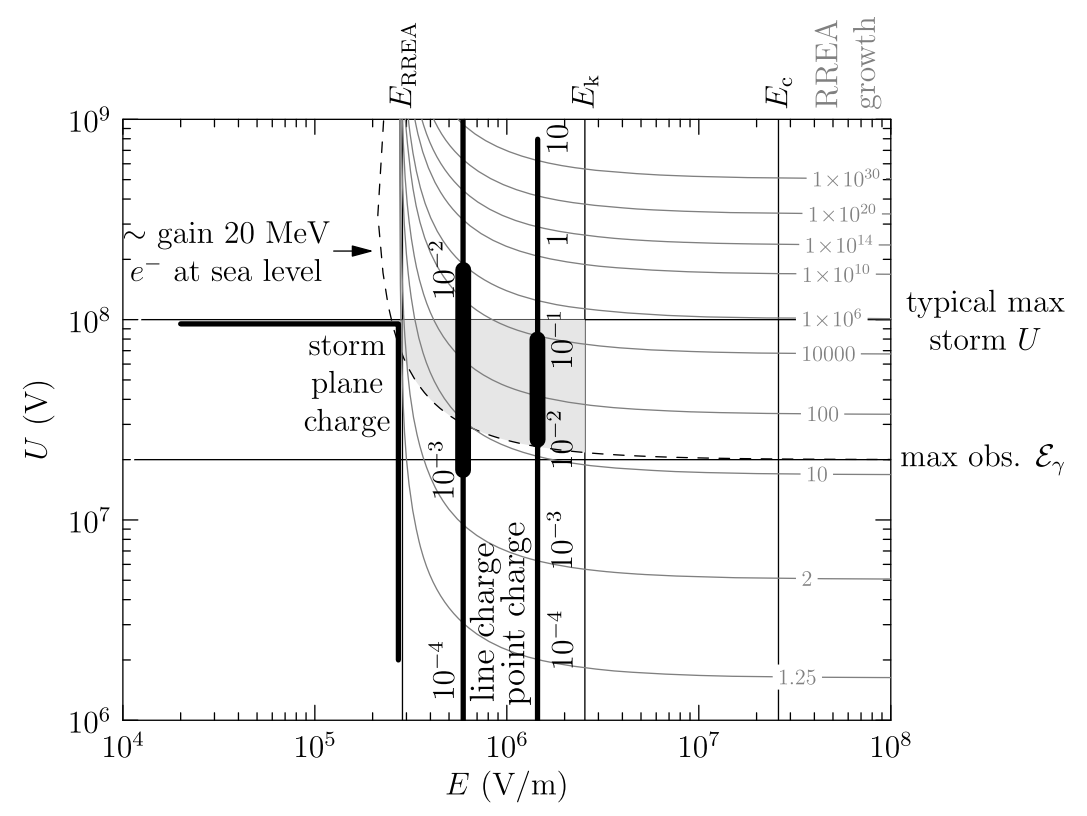

Figure 1. TGF and lightning constraints in voltage and electric field. The horizontal lines are reference voltages, while the vertical lines are reference electric fields as indicated. The dashed curve is the minimum voltage required for an energetic electron to gain $20 \mathrm{MeV}$ of energy in a given electric field in the presence of friction. The characteristics of point, line, and plane charges as discussed in the text are shown as labeled, with point and line charge magnitudes marked in $\mathrm{C}$ and $\mathrm{C} / \mathrm{m}$. Wide portions of the point and line contours indicate typical values inferred from lightning measurements. The maximum multiplication factor of RREA growth without relativistic feedback is shown as the grey contours. The TGF source voltage should not exceed the total maximum storm voltage but must exceed the voltage necessary to produce $20 \mathrm{MeV}$ electrons. The electric field must not exceed the conventional breakdown threshold over the large volumes necessary for TGF production but must exceed the RREA threshold to produce large populations of energetic electrons. The region satisfying these constraints is shaded grey. The overlap of the point and line charge characteristics with the grey region motivates our mechanism of leader-associated TGF production. 
Table 2. Typical Time Scales of Lightning Processes ${ }^{\mathrm{a}}$

\begin{tabular}{lc}
\hline \multicolumn{1}{c}{ Process } & Time Scale (ms) \\
\hline Streamer & 0.001 \\
HF pulses & 0.003 \\
Leader step & 0.003 \\
Narrow bipolar pulses & 0.01 \\
Return stroke & 0.1 \\
M components & 1 \\
K changes & 1 \\
Dart leader & 2 \\
Continuing current & 5 \\
Stepped leader growth & 30 \\
Return stroke separation & 50 \\
Lightning overall & few 100 \\
\hline
\end{tabular}

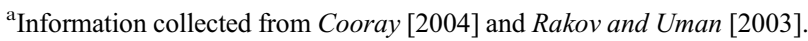

interested in producing large populations of energetic electrons, we focus on the region of space surrounding such charges where the electric field exceeds the RREA threshold. The length $(l)$ and total voltage available over that region $(U)$ can then be included in Figure 1 as $U$ and $\langle E\rangle \sim U / l$, giving the vertical lines marked as point and line charges. Typical lightning point charge (leader tip) and line charge (leader) values from Table 1 are indicated as wider lines.

\section{Reconciliation of Constraints}

[14] Lightning leaders and leader steps appear in the shaded region of Figure 1. These fields have sufficient average voltage and electric field to possibly produce TGFs.

[15] The idea that TGFs may be produced by fields near lightning leaders or leader steps is supported by the time scale of TGFs, a constraint we have not yet addressed. If TGFs are emitted on a time scale of $0.5 \mathrm{~ms}$, the process that produces them must produce a population of energetic electrons on such a time scale. Typical lightning process time scales are given in Table 2. Comparing the TGF time scale to the time scales of lightning processes, the best matches are current pulses along the lightning channel: return strokes, $\mathrm{K}$ changes, and $\mathrm{M}$ components. These redistributions of charge enhance the electric field around the leader channel, likely inducing leader stepping and streamer production/propagation. This electric field enhancement and leader activity occurs as the charges redistribute in the leader network with the time scale of the current pulse matching the TGF time scale. Multiple closely spaced current pulses such as $\mathrm{M}$ components may therefore explain multiple closely spaced gamma ray bursts. The local field enhancements caused by such current pulses would have similar parameters as typical leader and leader step processes, and therefore would fall in the same area of Figure 1.

[16] The remaining requirement is the production of $10^{17}$ energetic electrons. RREA processes alone can only amplify the number of particles by a factor of $10^{3}$ to $10^{5}$ for electric fields in this range, as shown in the grey contours in Figure 1 derived from RREA studies by Coleman and Dwyer [2006]. This process thus requires a seed population of $\geq 10^{12}$. Such large seed populations are very unlikely to be the result of cosmic rays due to the extreme rarity of sufficiently high-energy primary cosmic rays [Carlson et al., 2008]. One alternative is relativistic feedback where RREA effectively seeds itself such that a single initial energetic electron may suffice. This possibility has been discussed as relevant to TGF production by Dwyer [2008]. One difficulty of relativistic feedback as a seed source is that the field regions required are very large with total potentials approaching the maximum thunderstorm potential. This requirement limits the effectiveness of relativistic feedback in the confined electric fields we consider that surround leaders or leader tips. An alternative possibility that naturally arises near such breakdown is the production of energetic seed electrons by cold runaway in streamer tips.

[17] Recent measurements of energetic photons produced by sparks and streamers in the lab indicate the existence of electrons with energies in excess of $100 \mathrm{keV}$ [Nguyen et al., 2008; Rahman et al., 2008; Dwyer et al., 2008]. Such electrons have sufficiently high energy to serve as seed electrons for RREA. Similar results are also observed with lightning leaders [Dwyer et al., 2003, 2004]. Analytical estimates and detailed Monte Carlo calculations put the seed flux produced by an active leader tip at $\sim 10^{18} \mathrm{~s}^{-1}$, i.e., $10^{12}$ produced in a single $\sim 1 \mu$ s leader step [Moss et al., 2006; Gurevich et al., 2007]. These electrons are emitted in a region where the available voltage can accelerate them to high energies and the ambient electric field can result in RREA multiplication of the number of energetic particles. This process therefore may reach the required $10^{17}$ energetic electrons, especially if multiple regions throughout a leader network are driven simultaneously by a current pulse. The resulting population of energetic electrons can then produce bremsstrahlung observable as a TGF.

[18] This mechanism makes several predictions concerning TGF emission. First, TGFs should always be accompanied by lightning leaders. There are yet no clear TGF observations where coincident lightning is known not to have occurred, so this prediction is perhaps not surprising. Future lightning observations with receivers near and sensitive enough to detect the small impulses produced by weak intracloud lightning may settle this issue in the future. Second, TGFs should be observed in close time coincidence $(<1 \mathrm{~ms})$ with current pulses along leader channels, suggesting that radio emissions from the current pulse should be observed simultaneous with the TGF photon emission. This prediction has thus far been difficult to ascertain due to timing uncertainty in satellite data and the lack of available high-quality lightning information. Current TGF observations collected by the AGILE and Fermi satellites in conjunction with more modern lightning data may resolve this issue. Any TGFs observed without a causal lightning discharge or closely associated current pulse must be due to another mechanism. Finally, this mechanism implies energetic photons are emitted at the same altitude as lightning leaders and with a very wide directional distribution depending on the electric field structure. As existing models fit satellite data better with unphysical directional distributions manually broadened by up to $45^{\circ}$ [Carlson et al., 2007; Hazelton et al., 2009], naturally broad emissions as produced by this mechanism should be considered. Conversely, narrowly beamed emissions must be produced by a different mechanism.

[19] Finally, as this mechanism does not require extreme values of lightning parameters, we predict that energetic 
photon emission often accompanies lightning leaders, consistent with observations [Dwyer et al., 2003, 2004]. However, not all lightning discharges produce observable TGFs. First, note that not all lightning-induced gamma ray emission is observable by satellites. Low-altitude emissions are heavily attenuated and emissions of relatively few photons are difficult to distinguish from noise. The mechanism we discuss here may require uncommonly large current pulses through high-altitude leader channels to produce observable TGFs. Higher altitudes are indeed favored due to lower atmospheric density, lower frictional losses and correspondingly greater RREA growth rates, so low-altitude leaders would be less likely to produce the very intense high-energy photon pulses considered as TGFs. Second, as the global TGF frequency is not known, it may be the case that TGFs are far more common than existing measurements indicate. This will be tested in detail by the upcoming Airborne Detector for Energetic Lightning Emissions (ADELE), the Atmosphere Space Interactions Monitor (ASIM), and the Tool for the Analysis of Radiations from lightnings and Sprites (TARANIS).

\section{Summary}

[20] In this paper we first survey the requirements on TGF production: $15-20 \mathrm{~km}$ production altitude, $10^{17}$ energetic electrons, lightning electric fields that exceed the RREA threshold, and $20 \mathrm{MV}$ total available potential. We then survey lightning physics, extract constraints on the types of processes that can occur, and unify the constraints in Figure 1, leaving a small allowed region that produces enough high-energy photons without exceeding the breakdown threshold or the total available potential. These unified constraints suggest a mechanism driven by current pulses in lightning leaders and leader steps, naturally supported by the time scale of TGF emission. Our mechanism relies on cold runaway as a source of relativistic electrons, as suggested by observations of energetic photon emission in streamers, lab sparks, and in triggered lightning. The mechanism results in close time and space association between TGF emission and lightning leader current pulses which gives several testable predictions that should be observable in upcoming experiments.

[21] Acknowledgments. The authors would like to thank Ute Ebert and Thomas Gjesteland for useful discussions. This work was supported by the National Science Foundation under grant ATM-0535461.

[22] Zuyin Pu thanks the reviewers for their assistance in evaluating this paper.

\section{References}

Agostinelli, S., et al. (2003), G4-A simulation toolkit, Nucl. Instrum Methods Phys. Res., Sect. A, 506(3), 250-303.

Babich, L. P., R. I. Il'kaev, I. M. Kutsyk, A. Y. Kudryavtsev, R. A. RousselDupré, and E. M. Symbalisty (2004), Analysis of atmospheric gamma ray bursts based on the mechanism of generation of relativistic electron avalanches, Geomagn. Aeron., 44(2), 243-251.

Babich, L. P., E. N. Donskoy, I. M. Kutsyk, and R. A. Roussel-Dupré (2005), The feedback mechanism of runaway air breakdown, Geophys. Res. Lett., 32, L09809, doi:10.1029/2004GL021744.

Bazelian, E. M., and Y. P. Raizer (1998), Spark Discharge, CRC Press, Boca Raton, Fla.

Betz, H. D., U. Schumann, and P. Laroche (Eds.) (2009), Lightning. Principles, Instruments and Applications-Review of Modern Lightning Research, Springer, New York.
Carlson, B. E., N. G. Lehtinen, and U. S. Inan (2007), Constraints on terrestrial gamma ray flash production from satellite observation, Geophys. Res. Lett., 34, L08809, doi:10.1029/2006GL029229.

Carlson, B. E., N. G. Lehtinen, and U. S. Inan (2008), Runaway relativistic electron avalanche seeding in the Earth's atmosphere, J. Geophys. Res. 113, A10307, doi:10.1029/2008JA013210.

Cohen, M. B., U. S. Inan, and G. Fishman (2006), Terrestrial gamma ray flashes observed aboard the Compton Gamma Ray Observatory/Burst and Transient Source Experiment and ELF/VLF radio atmospherics, J. Geophys. Res., 111, D24109, doi:10.1029/2005JD006987.

Coleman, L. M., and J. R. Dwyer (2006), Propagation speed of runaway electron avalanches, Geophys. Res. Lett., 33, L11810, doi:10.1029/ 2006GL025863.

Cooray, V. (2004), The Lightning Flash, Inst. of Electr. Eng., London.

Cummer, S. A., Y. Zhai, W. Hu, D. M. Smith, L. I. Lopez, and M. A. Stanley (2005), Measurements and implications of the relationship between lightning and terrestrial gamma ray flashes, Geophys. Res. Lett., 32, L08811, doi:10.1029/2005GL022778.

Dwyer, J. R. (2003), A fundamental limit on electric fields in air, Geophys. Res. Lett., 30(20), 2055, doi:10.1029/2003GL017781.

Dwyer, J. R. (2008), Source mechanisms of terrestrial gamma-ray flashes, J. Geophys. Res., 113, D10103, doi:10.1029/2007JD009248.

Dwyer, J. R., and D. M. Smith (2005), A comparison between Monte Carlo simulations of runaway breakdown and terrestrial gamma-ray flash observations, Geophys. Res. Lett., 32, L22804, doi:10.1029/ 2005GL023848.

Dwyer, J. R., et al. (2003), Energetic radiation produced during rockettriggered lightning, Science, 299(5607), 694-697.

Dwyer, J. R., et al. (2004), A ground level gamma-ray burst observed in association with rocket-triggered lightning, Geophys. Res. Lett., 31 L05119, doi:10.1029/2003GL018771.

Dwyer, J. R., Z. Saleh, H. K. Rassoul, D. Concha, M. Rahman, V. Cooray, J. Jerauld, M. A. Uman, and V. A. Rakov (2008), A study of X-ray emission from laboratory sparks in air at atmospheric pressure, J. Geophys. Res., 113, D23207, doi:10.1029/2008JD010315.

Fishman, G. J., et al. (1994), Discovery of intense gamma-ray flashes of atmospheric origin, Science, 264(5163), 1313-1316.

Gallimberti, I., G. Bacchiega, A. Bondiou-Clergerie, and P. Lalande (2002), Fundamental processes in long air gap discharges, C. R. Phys., 3(10), $1335-1359$.

Grefenstette, B. W., D. M. Smith, J. R. Dwyer, and G. J. Fishman (2008), Time evolution of terrestrial gamma ray flashes, Geophys. Res. Lett., 35, L06802, doi:10.1029/2007GL032922.

Grefenstette, B. W., D. M. Smith, B. J. Hazelton, and L. I. Lopez (2009), First RHESSI terrestrial gamma ray flash catalog, J. Geophys. Res., 114, A02314, doi:10.1029/2008JA013721.

Gurevich, A. V., and K. P. Zybin (2001), Runaway breakdown and electric discharges in thunderstorms, Sov. Phys. Usp., Engl. Transl., 44(11), 1119-1140, doi:10.1070/PU2001v044n11ABEH000939.

Gurevich, A. V., K. P. Zybin, and Y. V. Medvedev (2007), Runaway breakdown in strong electric field as a source of terrestrial gamma flashes and gamma bursts in lightning leader steps, Phys. Lett. A, 361(1-2), 119-125.

Hazelton, B. J., B. W. Grefenstette, D. M. Smith, J. R. Dwyer, X.-M. Shao, S. A. Cummer, T. Chronis, E. H. Lay, and R. H. Holzworth (2009), Spectral dependence of terrestrial gamma-ray flashes on source distance, Geophys. Res. Lett., 36, L01108, doi:10.1029/2008GL035906.

Inan, U. S., and N. G. Lehtinen (2005), Production of terrestrial gamma-ray flashes by an electromagnetic pulse from a lightning return stroke, Geophys. Res. Lett., 32, L19818, doi:10.1029/2005GL023702.

Inan, U. S., M. B. Cohen, R. K. Said, D. M. Smith, and L. I. Lopez (2006), Terrestrial gamma ray flashes and lightning discharges, Geophys. Res. Lett., 33, L18802, doi:10.1029/2006GL027085.

Lehtinen, N. G., M. Walt, U. S. Inan, T. F. Bell, and V. P. Pasko (1996), $\gamma$-ray emission produced by a relativistic beam of runaway electrons accelerated by quasi-electrostatic thundercloud fields, Geophys. Res. Lett., 23(19), 2645-2648, doi:10.1029/96GL02573.

Lehtinen, N. G., T. F. Bell, and U. S. Inan (1999), Monte Carlo simulation of runaway $\mathrm{MeV}$ electron breakdown with application to red sprites and terrestrial gamma ray flashes, J. Geophys. Res., 104, 24,699-24,712, doi:10.1029/1999JA900335.

Marshall, T. C., and M. Stolzenburg (2001), Voltages inside and just above thunderstorms, J. Geophys. Res., 106, 4757-4768, doi:10.1029/ 2000JD900640.

Moss, G. D., V. P. Pasko, N. Liu, and G. Veronis (2006), Monte Carlo model for analysis of thermal runaway electrons in streamer tips in transient luminous events and streamer zones of lightning leaders, J. Geophys. Res., 111, A02307, doi:10.1029/2005JA011350.

Nemiroff, R. J., J. T. Bonnell, and J. P. Norris (1997), Temporal and spectral characteristics of terrestrial gamma flashes, J. Geophys. Res., 102, 96599666, doi:10.1029/96JA03107. 
Nguyen, C. V., A. P. J. van Deursen, and U. Ebert (2008), Multiple X-ray bursts from long discharges in air, J. Phys. D. Appl. Phys., 41(23), 234012, doi: $10.1088 / 0022-3727 / 41 / 23 / 234012$.

Østgaard, N., T. Gjesteland, J. Stadsnes, P. H. Connell, and B. Carlson (2008), Production altitude and time delays of the terrestrial gamma flashes: Revisiting the Burst and Transient Source Experiment spectra, J. Geophys. Res., 113, A02307, doi:10.1029/2007JA012618.

Rahman, M., V. Cooray, N. A. Ahmad, J. Nyberg, V. A. Rakov, and S. Sharma (2008), X rays from 80-cm long sparks in air, Geophys. Res. Lett., 35, L06805, doi:10.1029/2007GL032678.

Rakov, V., and M. Uman (2003), Lightning: Physics and Effects, Cambridge Univ. Press, Cambridge, U. K.

Roussel-Dupré, R. A., A. V. Gurevich, T. Tunnell, and G. M. Milikh (1994), Kinetic theory of runaway air breakdown, Phys. Rev. E, 49, 2257-2271, doi:10.1103/PhysRevE.49.2257.
Smith, D. M., L. I. Lopez, R. P. Lin, and C. P. Barrington-Leigh (2005), Terrestrial gamma flashes observed up to $20 \mathrm{MeV}$, Science, 307(5712), 1085-1088, doi:10.1126/science.1107466.

Stanley, M. A., X.-M. Shao, D. M. Smith, L. I. Lopez, M. B. Pongratz, J. D. Harlin, M. Stock, and A. Regan (2006), A link between terrestrial gamma-ray flashes and intracloud lightning discharges, Geophys. Res. Lett., 33, L06803, doi:10.1029/2005GL025537.

Williams, E., et al. (2006), Lightning flashes conducive to the production and escape of gamma radiation to space, J. Geophys. Res., 111, D16209, doi:10.1029/2005JD006447.

Wilson, C. T. R. (1924), The electric field of a thundercloud and some of its effects, Proc. Phys. Soc. London, 37, 32D-37D.

B. E. Carlson, U. S. Inan, and N. G. Lehtinen, Space, Telecommunications and Radioscience Laboratory, Electrical Engineering Department, Stanford University, Packard Bldg., 350 Serra Mall, Stanford, CA 94305-9515, USA. (brantc@stanford.edu) 